\title{
Fiducial markers for stereotactic lung radiation therapy: review of the transthoracic, endovascular and endobronchial approaches
}

\author{
Alessio Casutt $\mathbb{1}^{1,2}$, Rémy Kinj $\mathbb{1}^{2,3}$, Esat-Mahmut Ozsahin ${ }^{2,3}$, Christophe von Garnier ${ }^{1,2}$ and Alban Lovis ${ }^{1,2}$ \\ ${ }^{1}$ Division of Pulmonary Medicine, University Hospital of Lausanne, CHUV, Lausanne, Switzerland. ${ }^{2}$ University of Lausanne, UNIL, \\ Lausanne, Switzerland. ${ }^{3}$ Dept of Radiation Oncology, University Hospital of Lausanne, CHUV, Lausanne, Switzerland.
}

Corresponding author: Alessio Casutt (alessio.casutt@chuv.ch)

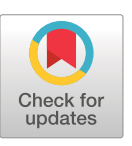

Copyright @The authors 2022

This version is distributed under the terms of the Creative Commons Attribution NonCommercial Licence 4.0. For commercial reproduction rights and permissions contact permissions@ersnet.org

This article has supplementary material available from err.ersjournals.com

Received: 29 June 2021 Accepted: 9 Sept 2021

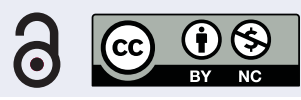

Shareable abstract (@ERSpublications)

Cyberknife allows accurate radiation delivery for inoperable peripheral pulmonary lesions by means of real-time tracking using fiducial markers (FMs). Coil-tailed and coil-spring FMs have shown the lowest migration rate with a high tracking rate. https://bit.ly/3CqM34T

Cite this article as: Casutt A, Kinj R, Ozsahin E-M, et al. Fiducial markers for stereotactic lung radiation therapy: review of the transthoracic, endovascular and endobronchial approaches. Eur Respir Rev 2022; 31: 210149 [DOI: 10.1183/16000617.0149-2021].

\section{Abstract}

Stereotactic body radiation therapy is an alternative to surgery for early-stage, inoperable peripheral nonsmall cell lung cancer. As opposed to linear accelerator (linac)-based (e.g. gating) and free-breathing techniques, CyberKnife ${ }^{\circledR}$ with Synchrony ${ }^{\circledR}$ technology allows accurate radiation delivery by means of a real-time respiratory motion tracking system using, in most cases, metal fiducial markers (FMs) placed in the vicinity of the target. The aims of this review are as follows. First, to describe the safety and efficacy of the transthoracic, endovascular and endobronchial FM insertion techniques for peripheral pulmonary lesions (PPLs). Second, to analyse performance in terms of the migration and tracking rates of different FM types. Recent developments in FM tracking for central lesions will also be reviewed. In conclusion, for PPLs, the endobronchial approach provides a low rate of pneumothorax, offers the possibility of concurrent diagnostic sampling for both the PPL and the lymph nodes, and, finally, reduces the intervention time compared to other techniques. In this context, coil-tailed and coil-spring FMs have shown the lowest migration rate with a consequently high tracking rate.

\section{Introduction}

The implementation of lung cancer screening programs applied to aged/smoker subjects using low-dose chest computed tomography (CT) scans will increase findings of peripheral pulmonary lesions (PPLs), stressing the need to develop their diagnostic and therapeutic management by promoting less invasive strategies. Stereotactic body radiation therapy (SBRT) is an alternative to surgery for early-stage, inoperable non-small cell lung cancer (NSCLC) or intrathoracic oligometastatic disease. As opposed to linear accelerator (linac)-based (e.g. gating) and free-breathing or breath-hold techniques, CyberKnife ${ }^{\circledR}$ with Synchrony ${ }^{\circledR}$ technology allows real-time respiratory motion tracking using, in most cases, dense metal (such as gold) fiducial markers (FMs) previously placed inside or near the tumour.

\section{Tracking versus non-tracking SBRT techniques}

Breath-induced motion is a major challenge when treating PPLs by SBRT. The average target motion in the superior-inferior direction has been measured to be up to $10-15 \mathrm{~mm}$, especially for lesions localised in the lower lobes [1].

Cyberknife ${ }^{\circledR}$ SBRT uses a 3D co-ordinate system that tracks the target using metallic FMs during the respiratory cycle in order to provide accurate non-coplanar radiation delivery. The tracking system permits a reduction in the margin from the macroscopic lesion to the planned-target volume and, therefore, enables a larger amount of healthy lung tissue surrounding the tumour to be spared [2, 3]. 
In contrast to non-tracking linac SBRT techniques, Cyberknife ${ }^{\circledR}$ has the advantage of being able to track the target co-ordinates in real time through non-coplanar irradiation, while other techniques require accelerator re-alignment to attenuate fluctuations of the target position. Moreover, this technique obviates the need for patients to perform prolonged apnoea, which is frequently unachievable for individuals with severe chronic obstructive pulmonary disease. Real-time target tracking by Cyberknife ${ }^{\circledR}$ can be achieved in two ways, i.e. by tracking the target itself (Xsight ${ }^{\circledR}$ Lung Tracking System, Accuray) or through previously inserted FMs. Xsight ${ }^{\circledR}$ Lung technology is based on the contrast difference between a solid target measuring at least $15 \mathrm{~mm}$ and the pulmonary parenchyma, but this condition is not met in most patients [4]. A flowchart of SBRT using Cyberknife ${ }^{\circledR}$ guided by FMs is shown in figure 1.

Compared to Cyberknife®, “breath-induced tumour motion” is managed using linac-based techniques with three main strategies:

1) The free-breathing technique consists of target irradiation for every possible position during the respiratory cycle using 4D-computed tomography. This technique increases the volume of healthy lung treated and the dose to planning target volume compared to the Cyberknife ${ }^{\circledR}$ technique $[5,6]$.

2) The gating technique consists of target irradiation during a specific respiratory phase (gate) during which the target motions are minimal. This technique has been improved with the development of real-time monitoring free-breathing systems such as the Varian Medical System (Palo Alto, USA). Compared to Cyberknife ${ }^{\circledR}$, gating techniques are time-consuming and lead to infra-fraction motions and shifts in the patient's position [7, 8].

3) The breath-hold technique consists of a temporary interruption of the patient's breathing. The patient breathes through a mouthpiece connected to an active breath control system. The respiratory signal is processed continuously using a monitor that displays the changing lung volume in real time. Irradiation can then be performed during apnoea, leading to a planning target volume reduction [9].

For central lesions (CLs), recent studies have shown that if "breath-induced tumour motion” is managed by linac-based techniques, SBRT can be safely delivered for both primary tumours or recurrence following surgery or conventional radiotherapy [10-13].

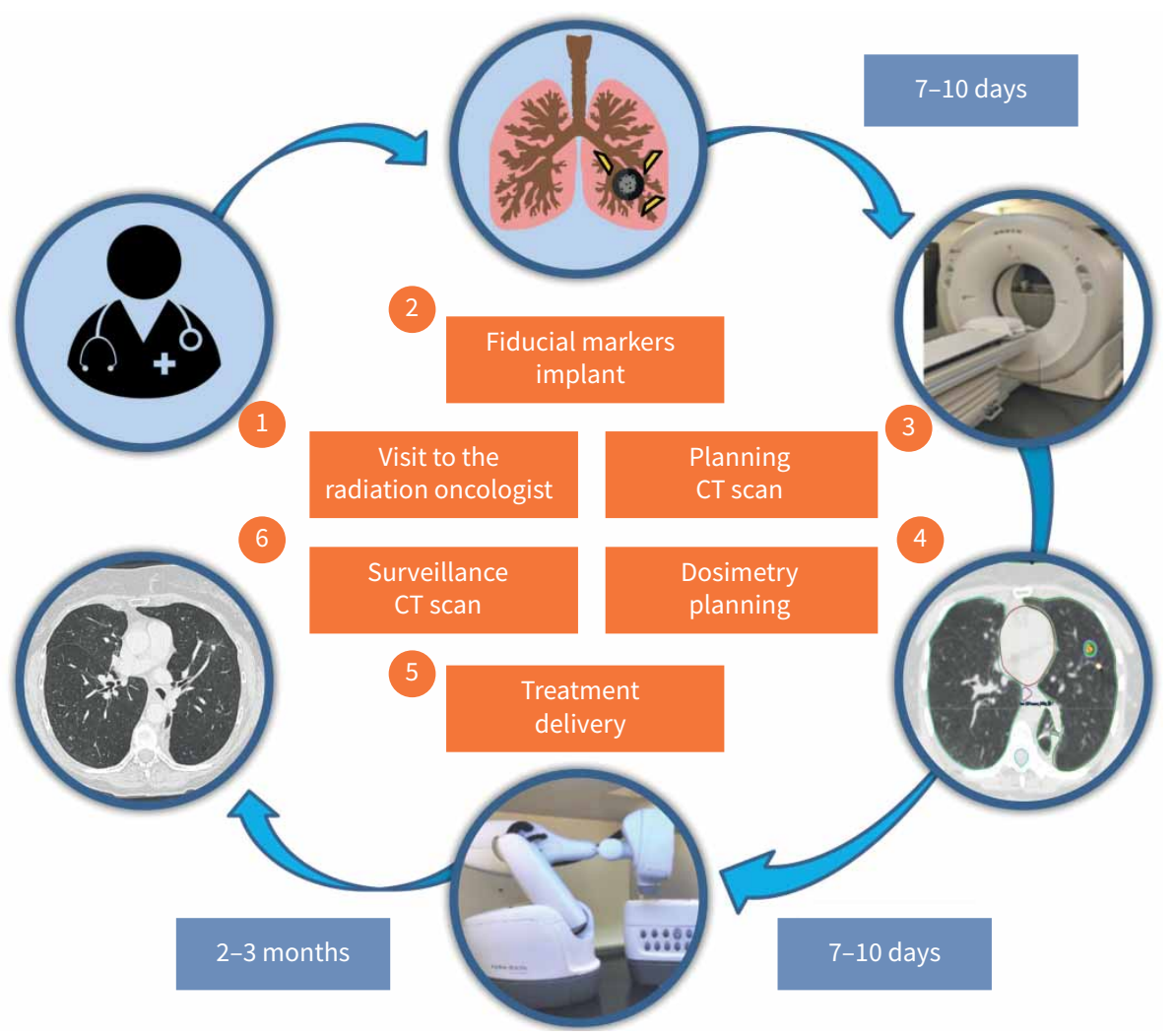

FIGURE 1 Flowchart of stereotactic lung radiation therapy using Cyberknife ${ }^{\circledR}$ guided by fiducial markers. 
Consequently, if Cyberknife ${ }^{\circledR}$ SBRT is applied to central lesions, breath-induced target motion should be taken into account and attenuated, as for PPL irradiation. This topic is well developed and summarised in the "state of the art" by Chang et al. [14] and in the review by BANG and BezJaK [15].

\section{PPLs and FMs}

The first case series description of FM placement for peripheral lesions reported the percutaneous (or transthoracic) insertion of linear gold FMs $(n=221)$ under CT scan in 48 patients [16]. Although widely available, this technique involves crossing the pleural surface in order to reach the target parenchymal lesion to insert FMs into/around the target lesion. In 2007, the authors described a pneumothorax rate of $33 \%(n=16)$. Subsequently, other comparable case series [17-20] have reported a similarly high rate of pneumothorax (38-67\%) [17-19], as summarised in supplementary table S1. The largest trials were published afterwards in 2014 [21] and 2017 [22] (sample size of 105 and 109 patients, respectively), and both studies reported a similarly high rate of pneumothorax (33\% and $43 \%$, respectively). The rate of target biopsy (transthoracic biopsy, TTBx) performed during the same procedure (before FM insertion) is poorly described in the literature but some small series with a TTBx reported rate of $0 \%$, described a similarly high rate of pneumothorax $(33-67 \%)[18,19,23]$ (supplementary table S1). Although this approach does not require general anaesthesia, the substantial risk of pneumothorax has attenuated its wider implementation; therefore, it is currently applied in most tertiary centres as a second-choice option.

Recently, the application of gelatine sponge slurry/powder or intra-parenchymal blood patching has led to a significantly lower pneumothorax rate (10\% [24], 11\%, [25] and 30\% [26]) and the need for chest tube placement [24-26]. Only one study has described FM percutaneous insertion associated with gelatine powder application and it showed a pneumothorax rate of 9\% [27]. Percutaneous FM insertion allows diagnostic sampling to be performed before FM insertion and enables management of endobronchial haemorrhage. In this context, if localised, pulmonary haemorrhage does not require specific treatment (16-30\%) [17, 21, 22, 27], whereas haemoptysis (0-4\%) [17, 21, 22, 27] may lead to additional radiological imaging, bronchoscopy or endovascular procedures.
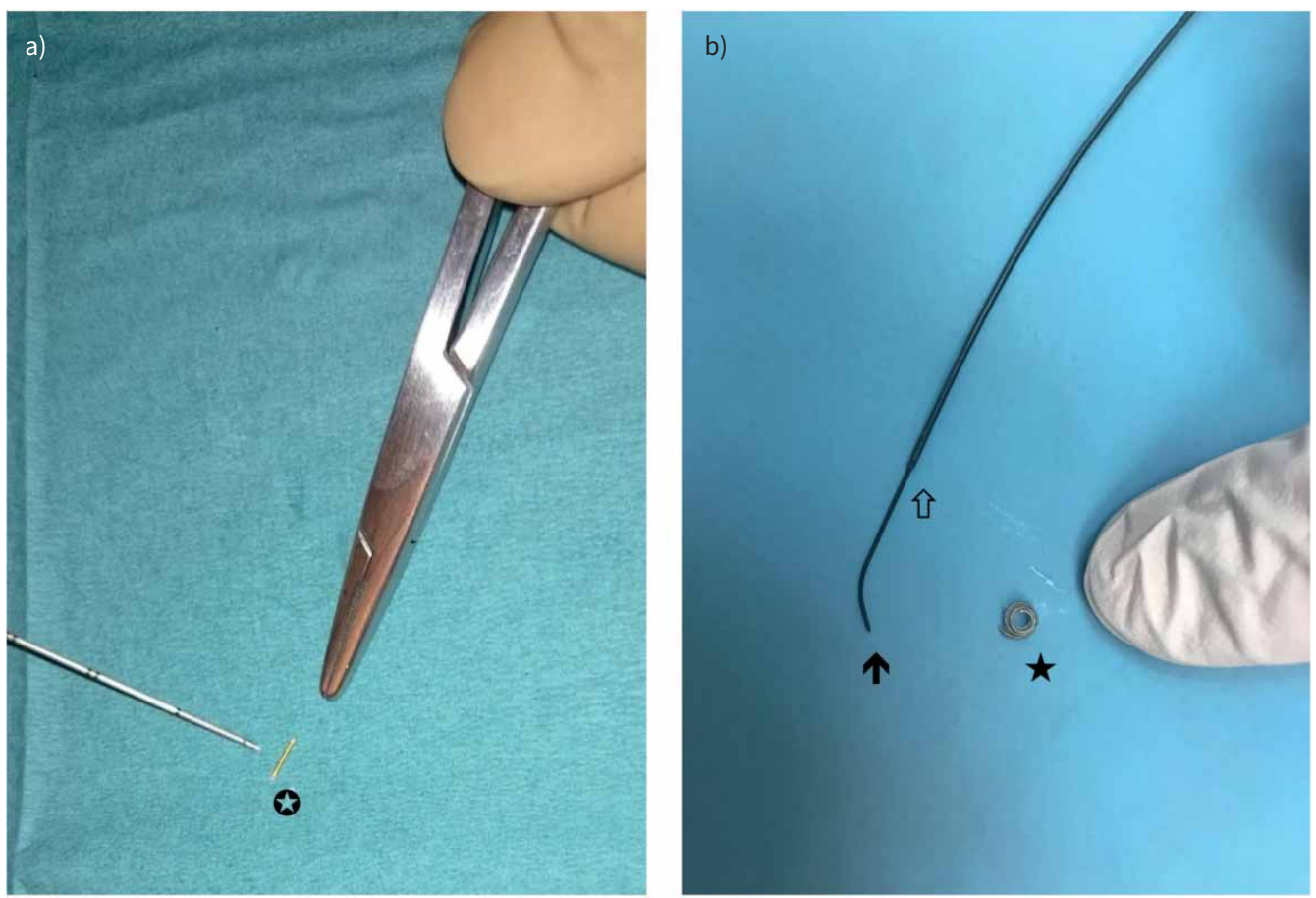

FIGURE 2 a) Linear gold $(5.0 \mathrm{~mm}$ length and $0.5 \mathrm{~mm}$ diameter) fiducial marker (white star; VisicoilTM IBA Dosimetry, Bartlett, TN, USA). Reproduced from [47] with permission. b) Coil-spring (diamond-shaped 3:2 mm) fiducial marker (black star; Tornado ${ }^{\oplus}$, Cook Medical, Bloomington, IN, USA). In the left part of the photograph, the distal part of the Progreat ${ }^{\circledast}$ (Terumo, Shibuya, Japan) guidewire (black arrow) inserted in the microcatheter (white arrow) can be seen. 
Furthermore, in cases of FM migration into the pleural space, the need for a second percutaneous procedure further increases the risk of complications. Two types of FM were described in this clinical context (figure 2), namely linear-FM and, recently, endovascular micro-embolisation coil FM (coil-spring FM, Cs-FM).

The FM migration rate was defined as the number of migrated FMs over the total number of inserted FMs. Migration was defined as an FM not being in the delivered and expected segment, or simply not found on the planning CT scan performed before SBRT delivery. Linear-FM use showed migration rates between 2 and $19 \%$ compared to $<1 \%$ for cs-FM [18-20, 22] insertion; however, the overall reported tracking rate is high at between 91 and 100\% [17-23] (supplementary table S1). The PPL tracking rate is defined as the number of PPLs tracked by Synchrony ${ }^{\circledR}$ or other software, over the total number of PPLs.

Concerning the median/mean procedure duration, no data have been reported in the literature. Finally, this approach is not applicable for CL targeting.

The endovascular approach respects lung anatomy by allowing the insertion of cs-FMs with a low $(<1 \%)$ pneumothorax risk (supplementary table S2). In 2008, Prévost et al. [28] provided the initial description of a selective catheterisation of sub-segmental pulmonary arteries close to the tumour via the right common femoral vein using angiography in 23 patients. The reported cs-FM migration rate was low (7\%), but non-coiling (defined by authors as non-complete winding thus without a complete rounded shape recovery) was frequent with a non-coiling rate at 29\%. Another small case series published in 2017 showed a similar migration rate of 8\% [22], and in 2019 BAKER et al. [23] reported the results of the largest trial in this field. The authors inserted 1335 cs-FMs in 416 patients, confirming the very high tracking rate of $100 \%[22,23,28]$ with this approach (supplementary table S2).

Although this technique is performed under local anaesthesia, it does not allow biopsies, requires venous access, a dedicated angiography infrastructure and a skilled operator, is time-intensive (mean procedure duration of $60 \mathrm{~min}$ [28]), is restricted to patients with sufficient kidney function, and is not suitable for CLs. These latter observations probably explain the limited uptake of this technique in tertiary centres.

The endobronchial approach for FM insertion similarly respects the natural airway pathway to pulmonary lesions. Consequently, the overall reported rate of complication is low compared to the percutaneous approach. Pneumothorax occurs in $0-6 \%$ [29-40] of cases and is dependent on the rate $(0-100 \%$ [30, 33-40]) of guided transbronchial biopsies. This technique, in contrast with the endovascular strategy, can be easily combined with the diagnostic sampling of PPL and lymph nodes. Moreover, this approach allows iatrogenic haemorrhage to be rapidly managed.

Initial reports of FM insertion guided by electromagnetic bronchoscopy (ENB) or radial-endobronchial ultrasound (R-EBUS) employed linear gold FMs. These were associated with a high migration rate of 10 $53 \%$ [29-32, 39, 41] (supplementary table S3) of the total number of inserted FMs and 18-20\% of treated PPLs [30, 42], with a tracking rate between 83 and 100\% [29-32].

Subsequently, non-linear FMs have been developed to optimise migration and tracking rates. FM types are schematised in figure 3.

In two studies, describing ENB-guided placement of nitinol wire attached to a gold seed (coil-tailed FM, ct-FM), CT scan simulation resulted in an FM migration rate of less than $1 \%$ and a tracking rate of $100 \%$
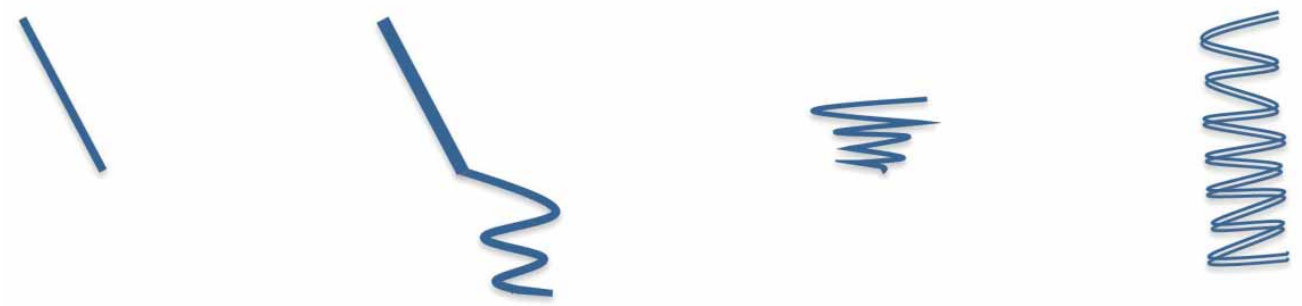

Linear FM

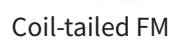

Coil-spring FM

Two-band FM

FIGURE 3 Fiducial marker (FM) types. 
$[35,43]$. Ct-FMs have been recently inserted by R-EBUS with encouraging results, i.e. a migration rate of $0 \%$ and a tracking rate of $99-100 \%[33,34]$.

A preliminary study described a multimodal guidance technique combining virtual bronchoscopy with ENB to place nine two-band FMs (SuperDimension ${ }^{\circledR}$ Covidien Inc, Minneapolis, MN, USA), and showed no migration [36].

So far, three studies have described ENB deployment of cs-FMs. They reported a migration rate between 1 and 3\% [38, 39, 41] and a high PPL tracking rate between 97 and 100\% [38, 39]. Endobronchial procedures performed with ENB require a dedicated, complex infrastructure, limiting its large-scale use, but they are timesaving compared to the endovascular approach, with a reported median procedure duration of $37 \mathrm{~min}$ [39]. However, moderate-to-deep sedation is needed to reduce motion once the patient is in the electromagnetic field, which may complicate the management of marginal patients.

We recently described our technique of cs-FM insertion under fluoroscopy guidance and assisted by R-EBUS [40]. In our study, we reported a migration rate of $8 \%$ and a tracking rate of $93 \%$, despite a lower PPL mean/median size (supplementary table S3). We also showed that previous conventional lung radiation therapy or SBRT in the PPL area was significantly associated with CS-FM migration. No other risk factor was identified in the literature. To reduce the migration rate of cs-FMs, a different approach, such as a larger FM device, may be required in previously irradiated lung areas with potentially distorted bronchi. Interestingly, only $2.4 \%$ of cs-FMs $(n=5)$ were incompletely deployed (non-coiling) at the planning CT scan. Despite such incomplete deployment or non-coiling, ineffective tracking occurred only in one PPL, representing one fifth of all PPLs with tracking failure. Our median procedure duration (32 min) was similar to the ENB-guided cs-FM placement technique. Reformatted planification CT scan images with a PPL surrounded by cs-FMs are shown in figure 4.

In this clinical setting, both ct-FMs and cs-FMs have shown the lowest migration rates leading to a high tracking rate and further prospective studies will be necessary to determine optimal endobronchial techniques.
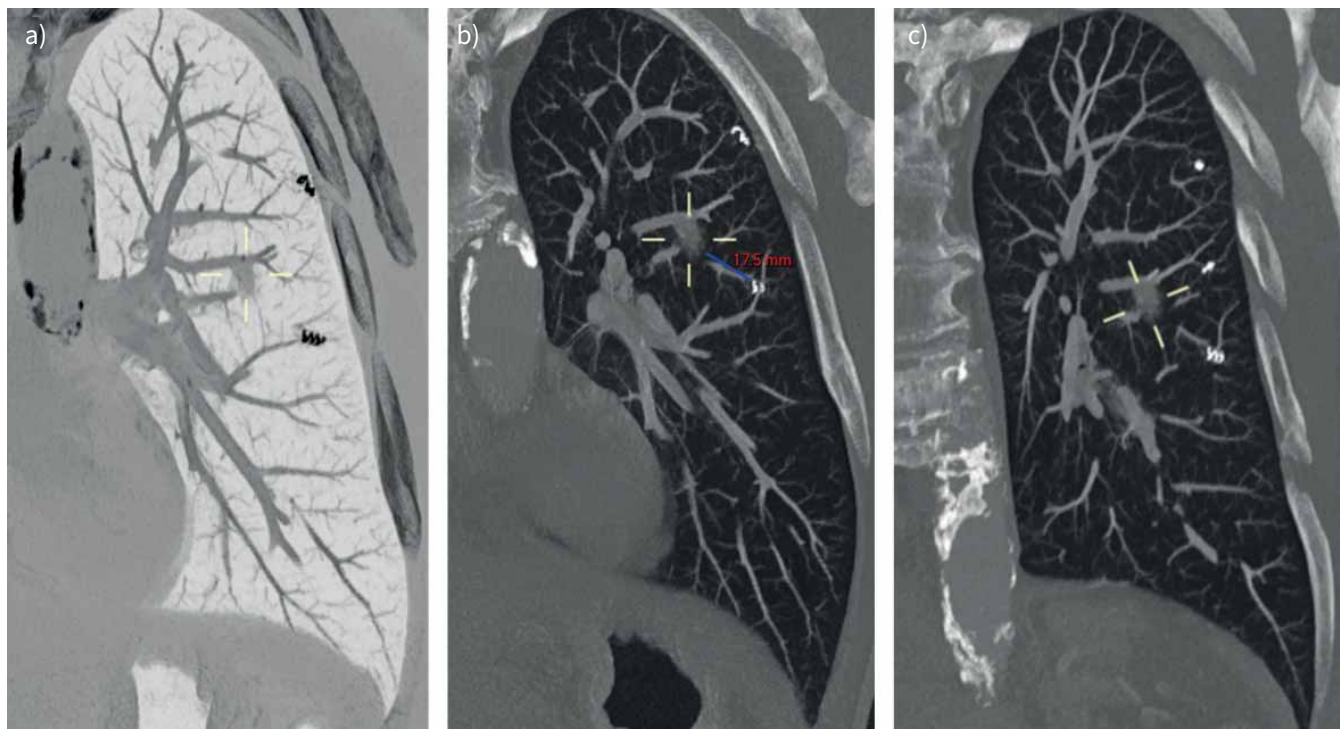

FIGURE 4 a) Reformatted planification computed tomography (CT) scan (performed before irradiation) image of lung windowing with maximum intensity projection (MIP) and an inverted-colour effect. The solid peripheral pulmonary lesion (PPL, measuring on long axis $11 \mathrm{~mm}, 440 \mathrm{~mm}^{3}$ ) is located the lateral segment of the left lower lobe and surrounded by two visible coil-spring fiducial markers (cs-FMs; diamond-shaped 3:2 mm; Tornado $^{\oplus}$, Cook Medical, Bloomington, IN, USA) in this oblique reformat. b) Reformatted planification CT scan (performed before irradiation) image of lung windowing with MIP of the same PPL. The minimal distance between the PPL and the border of the closest cs-FM is measured $(17.5 \mathrm{~mm})$. c) Reformatted planification CT scan (performed before irradiation) image of lung windowing with MIP of the same PPL. Three cs-FMs are identifiable in this oblique reformat. 


\section{CLs and FMs}

The implementation of SBRT techniques for CLs involving mediastinal or hilar lymph nodes has increased the utility of FM insertion in this clinical context [14, 15, 44]. The FM placement for CLs is particularly relevant due to the proximity of the main and lobar bronchi, the heart and the oesophagus. The radiation dose applied to the main and lobar bronchi is limited by major dose constraints [45]. Usually, treatment plans require a high dose gradient with a decrease of about $25 \%$ of dose in a few millimetres, while the average motion in the cranio-caudal direction for mediastinal stations is estimated to be about $1.24 \mathrm{~cm}$ [46].

Percutaneous and endovascular approaches are not applicable to CLs due to the excessive distance between the target and the pleura, as well as the large diameter of central pulmonary arteries.

Linear-endobronchial ultrasound (L-EBUS), known as convex-probe EBUS, is routinely used for the diagnosis of mediastinal mass and lymph nodes. In 2016, we described the safe insertion of a linear gold FM in a hilar lymph node by a transbronchial needle guided by L-EBUS [47]. Since then, case series have been described [48]. This approach requires deep sedation, specific equipment, an experienced operator and, furthermore, by crossing the bronchial wall for FM insertion into lymph nodes, this technique exposes the area to rare iatrogenic mediastinal infections [49].

In this setting, we recently described the endobronchial insertion of a rotatable clip (on quaternary carinas) normally used as a haemostatic option to manage gastrointestinal bleeding, potentially as a straightforward, non-invasive and rapid alternative [50].

In conclusion, the endobronchial approach provides a low rate of pneumothorax, is timesaving and offers the possibility of simultaneous diagnostic sampling of the target and lymph nodes. In this context, non-linear FM insertion guided by R-EBUS or ENB, namely ct-FM and cs-FM, have shown the lowest migration rates associated with high tracking rates. Further prospective studies are required to establish which endobronchial technique is optimal in a particular clinical setting. In our institution, a phase-III prospective randomised trial named "head-to-head comparison between endovascular cs-FMs and bronchoscopic cs-FMs insertion” is ongoing (clinicaltrials.gov: NCT04684186).

The implementation of SBRT techniques for CLs involving mediastinal or hilar lymph nodes has increased the utility of FM insertion in this clinical context. However, future trials are essential to provide robust data in such clinical situations.

Provenance: Submitted article, peer reviewed.

Conflict of interest: The authors have nothing to disclose.

\section{References}

$1 \quad$ Liu HH, Balter P, Tutt T, et al. Assessing respiration-induced tumor motion and internal target volume using four-dimensional computed tomography for radiotherapy lung cancer. Int J Radiat Oncol Biol Phys 2007; 68: 531-540.

2 Kilby W, Dooley JR, Kuduvalli G, et al. The Cyberknife robotic radiosurgery system in 2010. Technol Cancer Res Treat 2010; 9: 433-452.

3 El Shafie RA, Tonndorf-Martini E, Schmitt D, et al. Single-isocenter volumetric modulated arc therapy vs Cyberknife M6 for stereotactic radiosurgery of multiple brain metastases. Front Oncol 2020; 10: 568.

4 Bahig H, Campeau MP, Vu T, et al. Predictive parameters of Cyberknife fiducial-less (XSight Lung) applicability for treatment of early non-small cell lung cancer: a single-center experience. Int J Radiat Oncol Biol Phys 2013; 87: 583-589.

5 Prunaretty J, Boisselier P, Aillères N, et al. Tracking, gating, free-breathing, which technique to use for lung stereotactic treatments? A dosimetric comparison. Rep Pract Oncol Radiother 2019; 24: 97-104.

6 Ding $\mathrm{C}$, Chang $\mathrm{CH}$, Haslam J, et al. A dosimetric comparison of stereotactic body radiation therapy techniques for lung cancer: robotic versus conventional linac-based systems. J Appl Clin Med Phys 2010; 11: 3223.

7 Fox T, Simon EL, Elder E, et al. Free breathing gated delivery (FBGD) of lung radiation therapy: analysis of factors affecting clinical patient throughput. Lung Cancer 2007; 56: 69-75.

8 Purdie TG, Bissonnette JP, Franks K, et al. Cone-beam computed tomography for on-line image guidance of lung stereotactic radiotherapy: localization, verification, and intrafraction tumor motion. Int $J$ Radiat Oncol Biol Phys 2007; 68: 243-252. 
9 Wong JW, Sharpe MB, Jaffray DA, et al. The use of active breathing control (ABC) to reduce margin for breathing motion. Int J Radiat Oncol Biol Phys 1999; 44: 911-919.

10 Ceder YK, Fenig E, Popvtzer A, et al. Stereotactic body radiotherapy for central lung tumors, yes we can! Radiat Oncol 2018; $13: 77$.

11 Arnett ALH, Mou B, Owen D, et al. Long-term clinical outcomes and safety profile of SBRT for centrally located NSCLC. Adv Radiat Oncol 2019; 4: 422-428.

12 Nuyttens JJ, van der Voort van Zyp NC, Praag J, et al. Outcome of four-dimensional stereotactic radiotherapy for centrally located lung tumors. Radiother Oncol 2012; 102: 383-387.

13 Rowe BP, Boffa DJ, Wilson LD, et al. Stereotactic body radiotherapy for central lung tumors. J Thorac Oncol 2012; 7: 1394-1399.

14 Chang JY, Bezjak A, Mornex F, et al. Stereotactic ablative radiotherapy for centrally located early stage non-small-cell lung cancer: what we have learned. J Thorac Oncol 2015; 10: 577-585.

15 Bang A, Bezjak A. Stereotactic body radiotherapy for centrally located stage I non-small cell lung cancer. Transl Lung Cancer Res 2019; 8: 58-69.

16 Yousefi S, Collins BT, Reichner CA, et al. Complications of thoracic computed tomography-guided fiducial placement for the purpose of stereotactic body radiation therapy. Clin Lung Cancer 2007; 8: 252-256.

17 Kothary N, Heit JJ, Louie JD, et al. Safety and efficacy of percutaneous fiducial marker implantation for image-guided radiation therapy. J Vasc Interv Radiol 2009; 20: 235-239.

18 Bhagat N, Fidelman N, Durack JC, et al. Complications associated with the percutaneous insertion of fiducial markers in the thorax. Cardiovasc Intervent Radiol 2010; 33: 1186-1191.

19 Hong JC, Yu Y, Rao AK, et al. High retention and safety of percutaneously implanted endovascular embolization coils as fiducial markers for image-guided stereotactic ablative radiotherapy of pulmonary tumors. Int J Radiat Oncol Biol Phys 2011; 81: 85-90.

20 Hong JC, Eclov NCW, Yu Y, et al. Migration of implanted markers for image-guided lung tumor stereotactic ablative radiotherapy. J Appl Clin Med Phys 2013; 14: 4046.

21 Trumm CG, Häussler SM, Muacevic A, et al. CT fluoroscopy-guided percutaneous fiducial marker placement for CyberKnife stereotactic radiosurgery: technical results and complications in 222 consecutive procedures. J Vasc Interv Radiol 2014; 25: 760-768.

22 Mongeon M, Thibault F, Chartrand-Lefebvre C, et al. Safety and efficacy of endovascular fiducial marker insertion for Cyberknife stereotactic radiation therapy planning in early-stage lung cancer. $J$ Vasc Interv Radiol 2017; 28: 1090-1097.

23 Baker S, Sharma A, Antonisse I, et al. Endovascular coils as lung tumor fiducial markers for real-time tracking in stereotactic body radiotherapy: comparison of complication rates with transthoracic fiducial marker placement. J Vasc Interv Radiol 2019; 30: 1901-1907.

24 Renier H, Gérard L, Lamborelle P, et al. Efficacy of the tract embolization technique with gelatin sponge slurry to reduce pneumothorax and chest tube placement after percutaneous CT-guided lung biopsy. Cardiovasc Intervent Radiol 2020; 43: 597-603.

25 Perl RM, Risse E, Hetzel J, et al. The effect of intraparenchymal blood patching on the rate of pneumothorax in patients undergoing percutaneous CT-guided core biopsy of the lung. Eur J Radiol 2019; 116: 14-20.

26 Graffy P, Loomis SB, Pickhardt PJ, et al. Pulmonary intraparenchymal blood patching decreases the rate of pneumothorax-related complications following percutaneous CT-guided needle biopsy. J Vasc Interv Radiol 2017; 28: 608-613.e1.

27 Baadh AS, Hoffmann JC, Fadl A, et al. Utilization of the track embolization technique to improve the safety of percutaneous lung biopsy and/or fiducial marker placement. Clin Imaging 2016; 40: 1023-1028.

28 Prévost JB, Nuyttens JJ, Hoogeman MS, et al. Endovascular coils as lung tumor markers in real-time tumor tracking stereotactic radiotherapy: preliminary results. Eur Radiol 2008; 18: 1569-1576.

29 Anantham D, Feller-Kopman D, Shanmugham LN, et al. Electromagnetic navigation bronchoscopy-guided fiducial placement for robotic stereotactic radiosurgery of lung tumors: a feasibility study. Chest 2007; 132: 930-935.

30 Lachkar S, Guisier F, Roger M, et al. Assessment of per-endoscopic placement of fiducial gold markers for small peripheral lung nodules<20mm before stereotactic radiation therapy. Chest 2018; 153: 387-394.

31 Hagmeyer L, Priegnitz C, Kocher M, et al. Fiducial marker placement via conventional or electromagnetic navigation bronchoscopy (ENB): an interdisciplinary approach to the curative management of lung cancer. Clin Respir J 2016; 10: 291-297.

32 Harley DP, Krimsky WS, Sarkar S, et al. Fiducial marker placement using endobronchial ultrasound and navigational bronchoscopy for stereotactic radiosurgery: an alternative strategy. Ann Thorac Surg 2010; 89: 368-373.

33 Dobelbower MC, Popple RA, Minnich DJ, et al. Anchored transponder guided lung radiation therapy. Pract Radiat Oncol 2020; 10: e37-e44.

34 Lachkar S, Guisier F, Roger M, et al. A simple endoscopic method with radial endobronchial ultrasonography for low-migration rate coil-tailed fiducial marker placement. J Thorac Dis 2020; 12: 1467-1474. 
35 Belanger AR, Burks AC, Chambers DM, et al. Peripheral lung nodule diagnosis and fiducial marker placement using a novel tip-tracked electromagnetic navigation bronchoscopy system. J Bronchol Intervent Pulmonol 2019; 26: 41-48.

36 Steinfort DP, Siva S, Kron T, et al. Multimodality guidance for accurate bronchoscopic insertion of fiducial markers. J Thorac Oncol 2015; 10: 324-330.

37 Bowling MR, Folch EE, Khandhar SJ, et al. Fiducial marker placement with electromagnetic navigation bronchoscopy: a subgroup analysis of the prospective, multicenter NAVIGATE study. Ther Adv Respir Dis 2019; 13: 1753466619841234.

38 Nabavizadeh N, Zhang J, Elliott DA, et al. Electromagnetic navigational bronchoscopy-guided fiducial markers for lung stereotactic body radiation therapy: analysis of safety, feasibility, and interfraction stability. J Bronchol Interv Pulmonol 2014; 21: 123-130.

39 Schroeder C, Hejal R, Linden PA. Coil spring fiducial markers placed safely using navigation bronchoscopy in inoperable patients allows accurate delivery of CyberKnife stereotactic radiosurgery. $J$ Thorac Cardiovasc Surg 2010; 140: 1137-1142.

40 Casutt A, Noirez L, Bernasconi M, et al. Endobronchial coil spring fiducial markers for CyberKnife ${ }^{\circledR}$ stereotactic body radiation therapy. Respirol 2021; 26: 469-476.

41 Minnich DJ, Bryant AS, Wei B, et al. Retention rate of electromagnetic navigation bronchoscopic placed fiducial markers for lung radiosurgery. Ann Thorac Surg 2015; 100: 1163-1165.

42 Jackson P, Steinfort DP, Kron T, et al. Practical assessment of bronchoscopically inserted fiducial markers for image guidance in stereotactic lung radiotherapy. J Thorac Oncol 2016; 11: 1363-1368.

43 Rong Y, Bazan JG, Sekhon A, et al. Minimal inter-fractional fiducial migration during image-guided lung stereotactic body radiotherapy using SuperLock nitinol coil fiducial markers. PLOS ONE 2015; 10: e0131945.

44 Shepherd RW, Weiss E. Convex endobronchial utrasound-guided placement of fiducial markers in central lung tumors. J Bronchology Interv Pulmonol 2017; 24: e25-e26.

45 Hanna GG, Murray L, Patel R, et al. UK consensus on normal tissue dose constraints for stereotactic radiotherapy. Clin Oncol 2018; 30: 5-14.

46 Schaake EE, Belderbos JSA, Buikhuisen WA, et al. Mediastinal lymph node position variability in non-small cell lung cancer patients treated with radical irradiation. Radiother Oncol 2012; 105: 150-154.

47 Casutt A, Koutsokera A, Peguret N, et al. Linear-endobronchial utrasound-guided insertion of a fiducial marker: a new tool for tracking central lesions. J Bronchology Interv Pulmonol 2017; 24: 166-169.

48 Seides BJ, Egan JP, 3rd, French KD, et al. Fiducial marker placement for stereotactic body radiation therapy via convex probe endobronchial ultrasound: a case series and review of literature. $J$ Thorac Dis 2018; 10: 1972-1983.

49 Leong SC, Marshall HM, Bint M, et al. Mediastinal abscess after endobronchial ultrasound-guided transbronchial needle aspiration: a case report and literature review. J Bronchology Interv Pulmonol 2013; 20: 338-341.

50 Casutt A, Kinj R, Ozsahin EM, et al. Endobronchial clip device insertion for tracking central lesions. J Bronchol Interv Pulmonol 2022; in press [https://doi.org/10.1097/LBR.0000000000000787]. 